\title{
Gestational diabetes independently increases birth length and augments the effects of maternal BMI on birth weight: a retrospective cohort study
}

\section{Magdalena Byström ${ }^{1+}$, Anthony Liu ${ }^{1,2}{ }^{*}{ }^{\dagger}$, Ann Elizabeth Quinton ${ }^{1,2}$, Bernard Linton Champion $^{1,2}$, Kristy Mann 1, Michael Peek ${ }^{1,2}$ and Ralph Kay Heinrich Nanan ${ }^{1,2}$}

1 Sydney Medical School - Nepean, The University of Sydney, Penrith, NSW, Australia

${ }^{2}$ Charles Perkins Centre - Nepean, The University of Sydney, Penrith, NSW, Australia

Edited by:

Henry J. Rozycki, Children's Hospital

of Richmond at Virginia

Commonwealth University, USA

\section{Reviewed by:}

Megan Jane Wallace, MIMR-PHI

Institute and Monash University,

Australia

Sunita Pereira, Tufts Medical Center,

USA

\section{*Correspondence:}

Anthony Liu, Nepean Hospital, Level 5, South Block, Derby Street, Penrith, NSW 2751, Australia e-mail: anthony.liu@health.nsw. gov.au

${ }^{\dagger}$ Magdalena Byström and Anthony Liu have contributed equally to this work.
Objective:To investigate the effect of the interaction between gestational diabetes mellitus (GDM) and maternal body mass index (BMI) on the individual neonatal growth parameters.

Design: Retrospective cohort study.

Setting: A tertiary maternity service in Sydney, Australia, between 2005 and 2009.

Population: A cohort of 8859 women.

Methods: Generalized linear models.

Main outcome measures: Neonatal growth parameters, represented by z-scores for infant birth weight (BW), birth length (BL), and head circumference $(\mathrm{HC})$ in GDM and non-GDM groups.

Results: Only GDM alone had an independent and positive effect on BL $(p=0.02)$ but not on BW or HC. In addition, in pregnancies complicated with GDM, the association between maternal weight and BW was significantly stronger $(p<0.001)$. In combination, GDM and maternal BMI significantly affected $z$-score differences between BW and BL $(p<0.001)$, in that underweight mothers had babies that were lighter relative to their length and inversely obese mothers had babies that were heavier relative to their length.

Conclusion: GDM independently influences $B L$ and increases the association between maternal BMI and BW. In accordance with the hypothesis of the fetal origins of health and disease, the pronounced effects of GDM on fetal growth patterns demonstrated in this study are likely to influence long-term health outcomes in children.

Keywords: birth weight, birth length, head circumference, gestational diabetes mellitus, maternal BMI

\section{INTRODUCTION}

Fetal growth is determined by a complex interaction between genetic factors and the in utero environment (1). Changes in the in utero metabolic environment can be caused by both gestational diabetes mellitus (GDM) and maternal weight.

Effects on the fetus including those that effect growth are not only associated with short-term complications for the neonate but are also associated with health outcomes in later life. For example, the Dutch famine study demonstrated a clear link between fetal growth and a range of diseases, including cardiovascular disorders (2). This concept has been coined "The Developmental Origins of Health and Disease (DOHaD)" and was previously summarized under the Barker Hypothesis $(3,4)$.

A well established and important in utero determinant of birth weight (BW) is GDM (5). In this condition, insulin resistance and failure of $\beta$-cell compensatory mechanisms causes increased levels of maternal glucose and lipids. The elevated substrates are transported across the placenta overexposing the developing fetus to nutrients, causing an increase in fetal growth (6). GDM affects approximately $5-10 \%$ of pregnancies in Western nations (7).

Separate to GDM, maternal obesity may also be characterized by insulin resistance and elevated lipids, allowing excess nutrients to be transferred across the placenta to the fetus contributing to fetal growth (1). Maternal obesity has been described to have similar effect on BW as GDM $(8,9)$. Approximately onethird of pregnancies are affected by maternal obesity, and in the female Australian population 2011-2012, approximately $56 \%$ were classified as overweight or obese $(1,10)$.

Apart from BW, fetal growth can also be determined by birth length (BL) and head circumference (HC). To be able to describe growth in more detail, it is also important to acknowledge the relationship of these parameters to each other as a measure of fetal growth.

Therefore, the aim of this study was to investigate the effect of the interaction between GDM and maternal body mass index 
(BMI) on individual neonatal growth parameters and their relationship to each other.

\section{MATERIALS AND METHODS}

This is a retrospective cohort study of deliveries from a tertiary maternity service in Metropolitan Sydney, New South Wales, Australia, occurring between 2005 and 2009. Women were identified using the hospital's obstetric database. The database is updated and maintained during and after pregnancy, and contributes to statewide data collection. The database identified 18,304 pregnancies, of which all live-born and singleton pregnancies with gestational age greater than 24 weeks were eligible for inclusion in the study (Figure 1). However, cases were excluded if the data were incomplete for maternal and neonatal anthropometric measurements or contained data entry errors $(n=9235)$. Mothers with pre-existing diabetes were further excluded from the study $(n=41)$.

Maternal characteristics extracted from the database included weight and height measured at the booking visit, age, parity, smoking status, and obstetric complications including GDM and pre-eclampsia. Maternal BMI, calculated as $\mathrm{kg} / \mathrm{m}^{2}$, were divided into six BMI ranges according to the WHO-criteria: underweight $\left(<18.5 \mathrm{~kg} / \mathrm{m}^{2}\right)$, normal weight $\left(18.5-24.9 \mathrm{~kg} / \mathrm{m}^{2}\right)$, overweight $\left(25-29.9 \mathrm{~kg} / \mathrm{m}^{2}\right)$, obese class I (30-34.9 $\left.\mathrm{kg} / \mathrm{m}^{2}\right)$, obese class II $\left(35-39.9 \mathrm{~kg} / \mathrm{m}^{2}\right)$, and obese class III $\left(\geq 40 \mathrm{~kg} / \mathrm{m}^{2}\right)(11)$. Maternal smoking was self-reported and included smoking at any time during pregnancy.

All pregnant women attending the antenatal clinic were offered screening for GDM, which was done at 24-28 weeks of gestation. Depending on the medical or family history, a $50 \mathrm{~g}$ glucose challenge test (GCT) or a $75 \mathrm{~g} 2 \mathrm{~h}$ oral glucose tolerance test (OGTT) was conducted. Women were considered high risk for GDM if the 1-h GCT was $>7.8 \mathrm{mmol} / \mathrm{L}$ with the diagnosis being confirmed by subsequent $2 \mathrm{~h}$ OGTT. GDM was diagnosed based upon contemporaneous Australasian Diabetes in Pregnancy Society (ADIPS) criteria; fasting glucose during OGTT $\geq 5.5 \mathrm{mmol} / \mathrm{L}$ or 2-h glucose was $\geq 8.0 \mathrm{mmol} / \mathrm{L}$ (12).

Neonatal variables extracted from the database included gestational age at birth, BW, BL, and HC. Gestational age, in weeks, was estimated from the time of the last menstrual period (LMP), and was confirmed by ultrasonography. If there was a greater than 10-day difference between the estimated age by LMP and the estimated age on second trimester ultrasonography, the age calculated by ultrasonography was used.

$z$-scores, adjusted for gestational age, were calculated for BW, $\mathrm{BL}$, and $\mathrm{HC}$ without customizing for maternal characteristics (13).

\section{STUDIED OUTCOMES}

The outcomes studied were neonatal growth variables, represented by gestational age adjusted $z$-scores for infant BW, BL, and $\mathrm{HC}$. To exclude biologically unrealistic values, the outcome variables were further restricted; infants were excluded from the analyses if any of the $z$-scores were $>3$ or $<-3$ standard deviations from the expected mean $(n=169)$. This was applied to avoid outliers in documentation or potential other pathologies either not or inaccurately listed in the database.

To obtain the neonatal growth proportionality, the difference between the different $z$-scores was calculated: 1: HC minus BW $(\mathrm{HC}-\mathrm{BW}) ; 2$ : $\mathrm{BW}$ minus $\mathrm{BL}(\mathrm{BW}-\mathrm{BL})$; and 3: $\mathrm{HC}$ minus $\mathrm{BL}$ $(\mathrm{HC}-\mathrm{BL})$. For example, a baby might have a BW $z$-score of +1 $(+1$ SD from its expected mean), but a HC of -1 ( -1 SD from its expected mean), which results in a difference of 2 between the two outcome variables, which would be a surrogate for an asymmetric growth pattern. A perfect symmetric growth pattern would result in a difference of 0 .

\section{STATISTICS}

Descriptive statistics are reported as mean $\pm \mathrm{SD}$ and median and interquartile range. Maternal characteristics and pregnancy

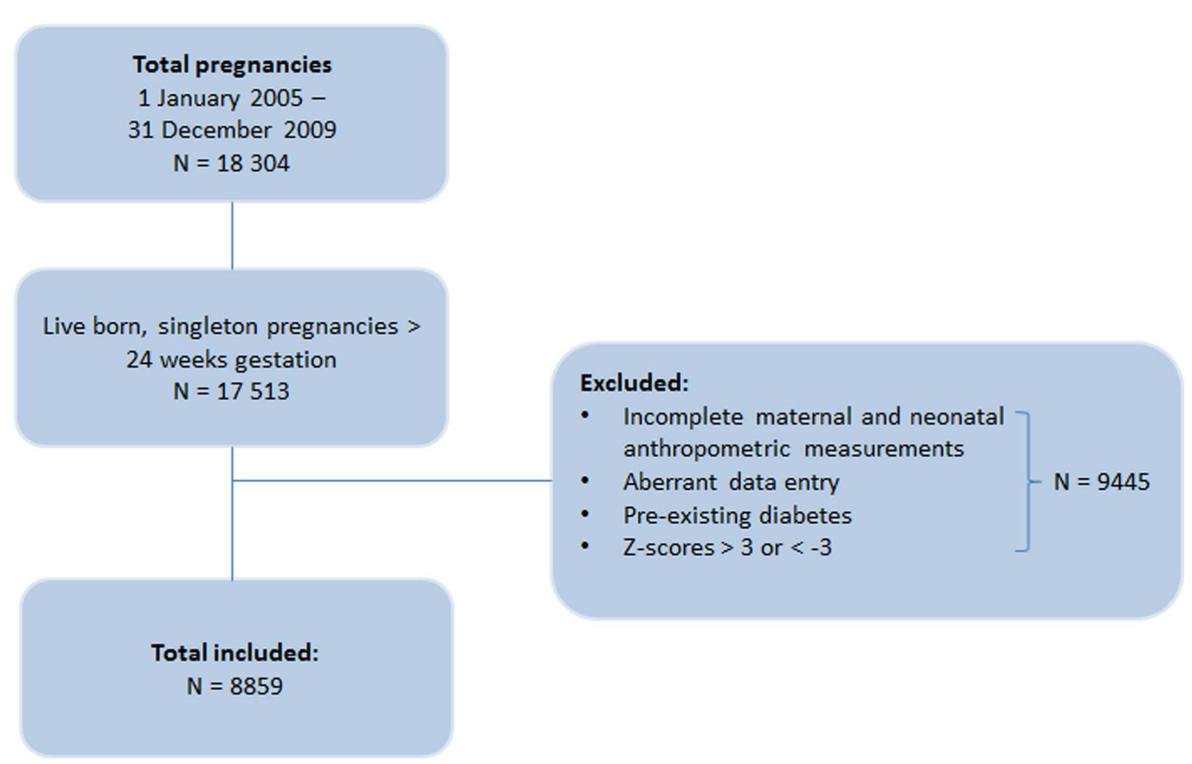

FIGURE 1 | Flow chart of the inclusion of pregnancies in the study 
outcomes were compared between GDM and non-GDM women using a Students $t$-test for normally distributed continuous data, a Mann-Whitney $U$-test for skewed continuous data or a chi-squared test for categorical data.

Generalized linear models were used to examine the effect of GDM (yes/no) on the studied outcomes. The models were adjusted for maternal age, maternal BMI (categorical), parity, smoking (yes/no), maternal pre-eclampsia and hypertension, gestational age, and infant gender. Additionally, an interaction term between GDM and maternal BMI was investigated in each of the outcome models. The adjusted means in each GDM group from these models were plotted over the maternal BMI categories.

Table 1 | Maternal characteristics and pregnancy outcomes in GDM and non-GDM women.

\begin{tabular}{lccc}
\hline Characteristics & GDM $(\boldsymbol{n}=\mathbf{4 9 8})$ & $\begin{array}{c}\text { Non-GDM } \\
(\boldsymbol{n}=\mathbf{8 3 6 1})\end{array}$ & $\boldsymbol{p}$ value \\
\hline Maternal age (years) & $31.6 \pm 5.2$ & $28.6 \pm 5.3$ & $<0.001$ \\
Maternal BMI (kg/m²) & $32.0[24.3-35.0]$ & $24.8[21.5-29.7]$ & $<0.001$ \\
Parity & $1.0[1.0-2.0]$ & $1.0[1.0-2.0]$ & 0.034 \\
Smoking (\%) & $99(19.9)$ & $2208(26.4)$ & 0.002 \\
Hypertension (\%) & $40(8)$ & $418(5)$ & 0.004 \\
Pre-eclampsia (\%) & $13(2.6)$ & $163(2)$ & 0.4 \\
Gestational age (weeks) & $39.0[38.2-39.5]$ & $39.5[38.6-40.4]$ & $<0.001$ \\
Birth weight (g) & $3384 \pm 532$ & $3455 \pm 521$ & 0.003 \\
Birth length (cm) & $51.0[49.0-53.0]$ & $51.0[49.5-53.0]$ & 0.5 \\
Head circumference (cm) & $34.5[33.5-35.5]$ & $34.5[33.5-35.5]$ & 0.9 \\
BW z-score & $0.29 \pm 1.1$ & $0.09 \pm 1.0$ & $<0.001$ \\
BL z-score & $0.18 \pm 1.0$ & $-0.02 \pm 0.94$ & $<0.001$ \\
HC z-score & $0.12 \pm 0.89$ & $-0.04 \pm 0.86$ & $<0.001$ \\
\hline
\end{tabular}

Values are shown as mean $\pm S D$, median [01-03] or $n(\%)$.

$\mathrm{cm}$, centimeter.
SAS Version 9.3 software (SAS Institute Inc., Cary, NC, USA) was used for all statistical analyses. A $p$-value of $<0.05$ was considered statistically significant. No adjustment was made for multiple comparisons.

\section{ETHICS}

The study was approved by the Local Human Research Ethics Committee of the Nepean Blue Mountains Local Health District on 18 March 2013 (HREC no. 10/16).

\section{RESULTS}

\section{DEMOGRAPHICS}

The study included 8859 women, of whom 498 (5.6\%) patients had GDM. Maternal characteristics and pregnancy outcomes for GDM and non-GDM women are shown in Table 1.

In pregnancies complicated with GDM, mothers were older and had higher average BMI. They were also less likely to smoke. Examining the $z$-scores, adjusted for gestational age, there were significant differences for BW, BL, and $\mathrm{HC}$ between the two groups, where GDM women gave birth to babies that were heavier, longer, and had greater HC compared to babies born to non-GDM women. Table 2 summarizes birth parameters for all BMI groups.

\section{THE INDEPENDENT EFFECT OF GESTATIONAL DIABETES ON GROWTH}

Examining the independent effect of GDM on the neonatal growth variables, i.e., $z$-scores for $\mathrm{BW}, \mathrm{BL}$, and $\mathrm{HC}$, models adjusted for maternal age, maternal BMI, parity, smoking status, hypertension, pre-eclampsia, gestational age, and infant gender, were used. The results show that GDM only had an independent effect on BL $z$ score $(p=0.02)$. Excluding GDM, there is an independent effect of BMI on $z$-scores for BW, BL, and $\mathrm{HC}(p<0.001)$.

\section{THE INTERACTION EFFECT BETWEEN GDM AND MATERNAL BMI}

The interaction effect between GDM and maternal BMI on the neonatal growth variables was examined. Figure 2 compares the association between increasing maternal BMI and BW $z$-score in women with and without GDM after adjusting for all confounding

Table 2 | Birth parameters according to BMI categories.

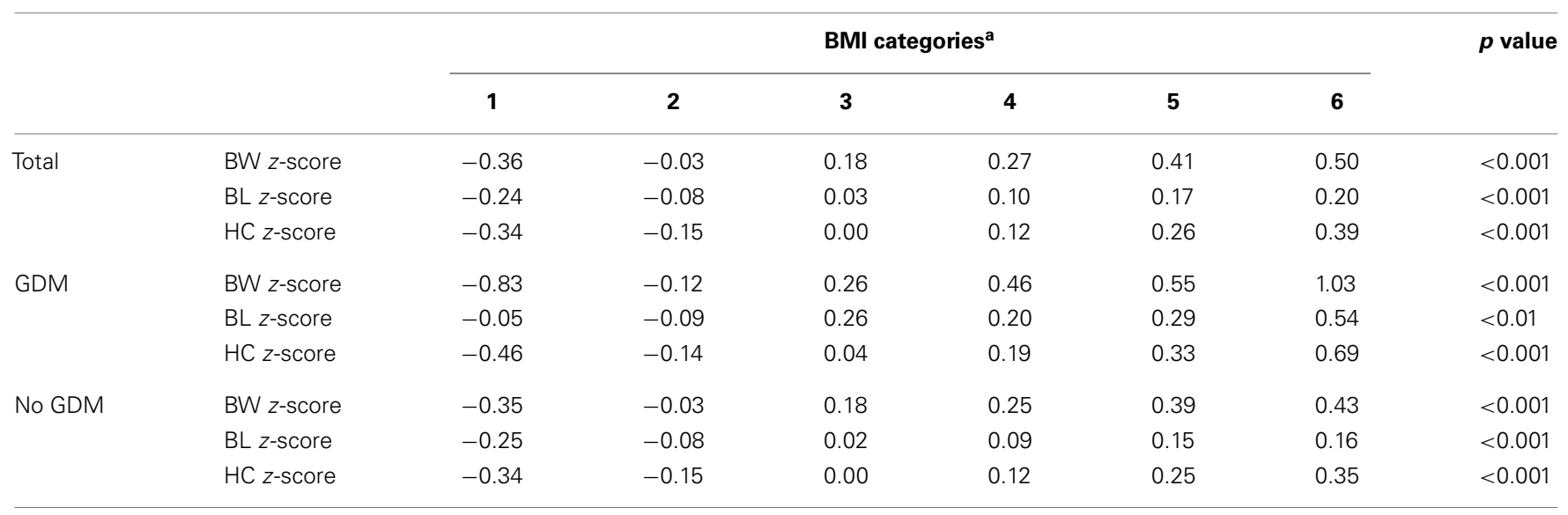

z-scores reported in means.

$B W$, birth weight; $B L$, birth length; $H C$, head circumference.

${ }^{a}$ BMl category $1:<18.5 \mathrm{~kg} / \mathrm{m}^{2} ; 2: 18.5-24.9 \mathrm{~kg} / \mathrm{m}^{2} ; 3: 25-29.9 \mathrm{~kg} / \mathrm{m}^{2} ; 4: 30-34.9 \mathrm{~kg} / \mathrm{m}^{2} ; 5: 35-39.9 \mathrm{~kg} / \mathrm{m}^{2} ; 6: \geq 40 \mathrm{~kg} / \mathrm{m}^{2}$. 


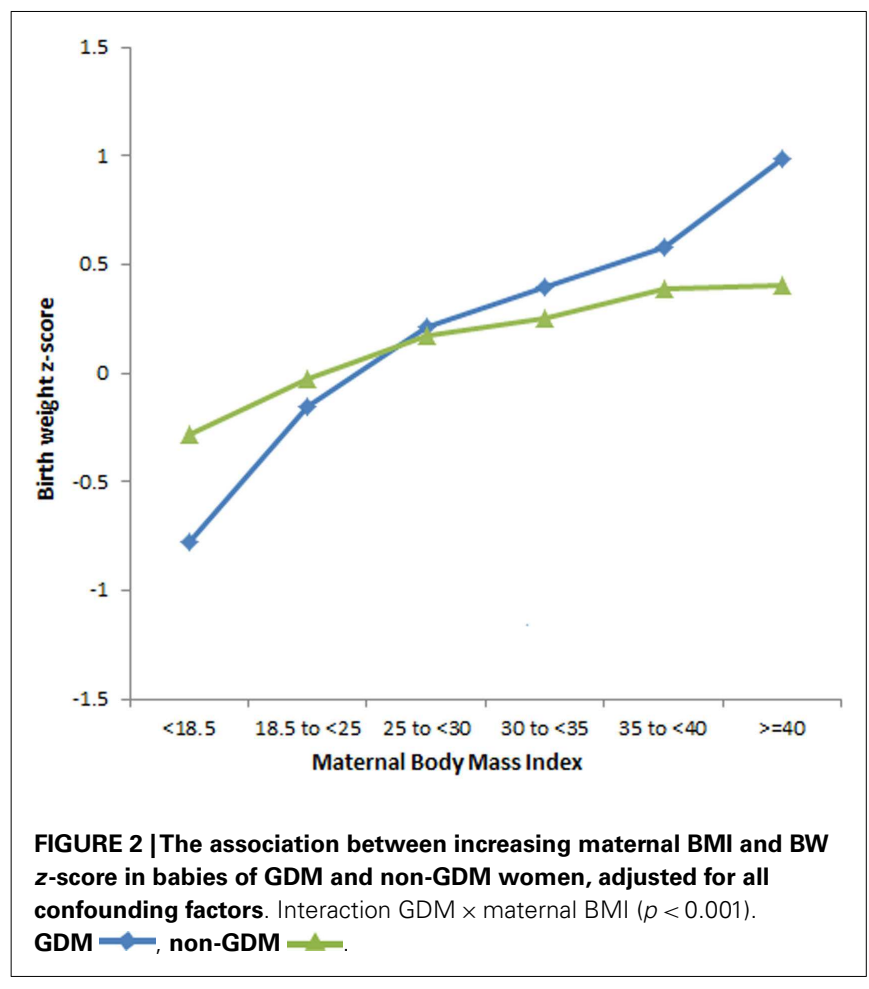

factors. The increase in BW with increasing maternal BMI is more pronounced in pregnancies with GDM than in non-GDM pregnancies $(p<0.001)$.

There was no interaction effect between maternal BMI and GDM on BL $z$-scores $(p=0.19)$ and HC $z$-scores $(p=0.27)$.

\section{EFFECT OF GDM ON INTRA-INDIVIDUAL LENGTH AND WEIGHT Z-SCORES}

As we have described an independent effect of GDM on length $z$-scores and a combined effect of GDM and maternal BMI on weight $z$-score, we examined the effect of GDM on the relationship between weight and length $z$-scores (Figure 3). In GDM women with $\mathrm{BMI}<18.5$, the growth asymmetry is enhanced with babies having a mean difference of almost 0.74 between their BW and BL $z$-scores. The interaction effect of maternal BMI and GDM on the relationship between BW and BL $z$-scores (BW-BL) is highly significant $(p<0.001)$.

\section{DISCUSSION}

\section{MAIN FINDINGS}

This retrospective study has confirmed that the association between maternal weight and BW is more pronounced in pregnancies complicated with GDM. We have also found that GDM together with maternal BMI affects the neonatal growth patterns, specifically the relationship between BW and BL. Lean GDM women gave birth to babies with less weight compared to their length. Conversely, obese GDM women gave birth to babies with greater weight compared to their length. Since the interaction between maternal BMI and GDM did not have an effect on the $\mathrm{HC} z$-score or the BL $z$-score, it is proposed that the variation in $\mathrm{BW}$, in relation to maternal BMI, contributes to the asymmetry

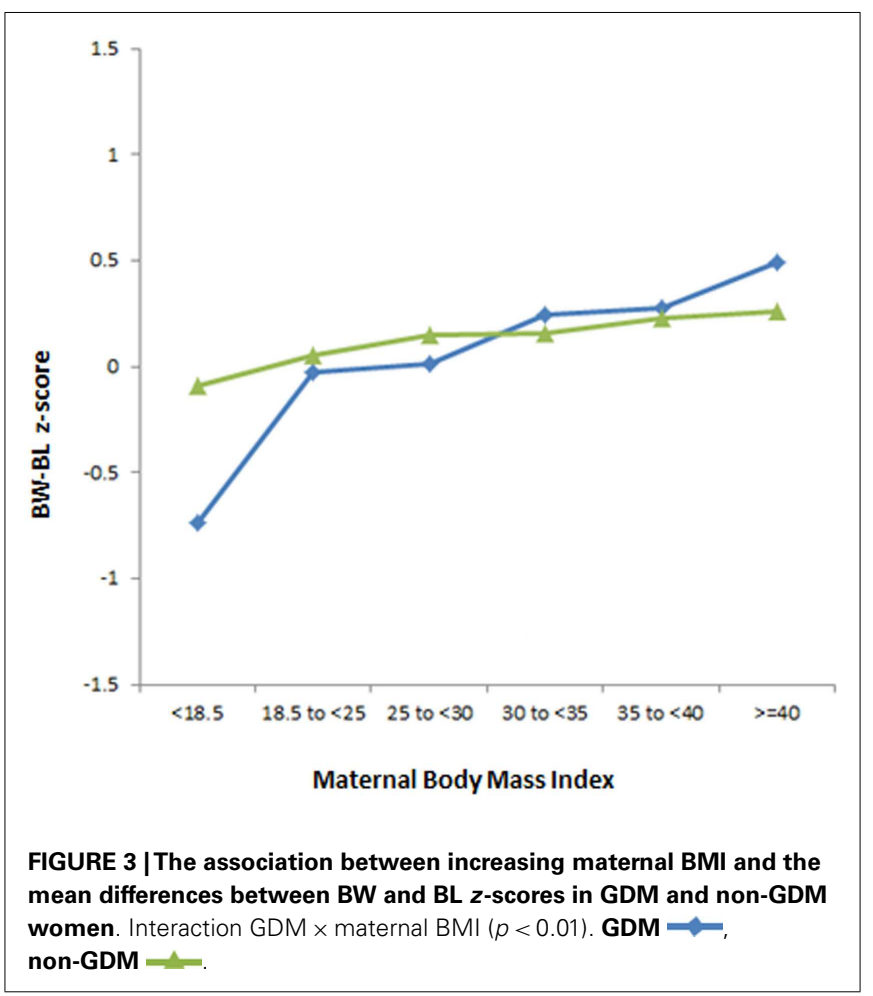

among babies born to GDM women. In summary, the interaction between maternal weight and GDM does not seem to affect skeletal growth, only fetal weight.

\section{STRENGTHS AND LIMITATIONS}

The strength of our study is that this study has systematically compared the symmetry between BW, BL, and HC, between controls and pregnancies affected by GDM in relation to increasing maternal BMI.

Limitations to this study are its retrospective nature and the unavailability of measures of maternal blood glucose values, which have been shown to correlate to fetal and/or neonatal growth patterns (14). In addition, the effect of treatment modality on different growth parameters and growth proportion would be interesting to investigate. Furthermore, a potential confounding factor is that women with GDM were usually delivered electively at 38 weeks, and hence the natural progression of fetal proportions in these women beyond this gestational age could not be ascertained. Another area to consider is that we used booking weight (BMI) in our study. Since weight gain during pregnancy has been found to affect BW, it is possible that weight gain/loss in pregnancy might have affected our results (15). Unfortunately, our data collection system does not capture these parameters.

\section{INTERPRETATION}

The effect of GDM on BW has been demonstrated in previous studies $(7,16)$. In addition, the HAPO study found a strong and continuous correlation between maternal glucose levels and increased BW (5). Although our results showed that GDM did not have a significant independent effect on BW, we found that GDM did enhance the effect of maternal BMI on BW confirming 
previous observations $(17,18)$. Makgoba et al. investigated the interaction between GDM and maternal BMI on BW in white, black, and South Asian women. They found that the enhancing effect of GDM on the association between maternal BMI and BW differed between the racial groups, suggesting that part of the interaction effect between GDM and maternal BMI can be attributed to genetic factors (17). The vast majority of the cohort in this study was of Caucasian origin.

This interaction effect cannot completely be explained by genetics. Women who develop GDM show resistance to the action of insulin to stimulate glucose disposal and to suppress production of glucose and fatty acids, resulting in increased maternal plasma glucose levels (7). The exposure to increased maternal glucose supply stimulates fetal pancreatic insulin production, leading to accelerated fetal growth (19). However, circulating levels of other nutrients, such as triglycerides (TG) and free fatty acids (FFA), are also increased in GDM and may also contribute to fetal growth (1, $6,20)$. In GDM, other alterations affecting fetal growth have been found, including increased placental weights, which could increase placental nutrient transfer, thereby increasing fetal nutrient supply (21). Other alterations include altered levels of adipokines correlated to BW $(20,22)$.

Maternal obesity, in the absence of GDM, has been found to have a strong independent relationship with BW and the pathophysiology may have similarities with GDM (8). Adipose tissue was originally considered to be a storage site for TG. However, adipose tissue is metabolically active with multiple endocrine and immune functions, with the potential to produce disturbances in insulin signaling pathways resulting in increased insulin resistance with increasing weight (19). Maternal obesity is also characterized by dyslipidaemia, which, together with insulin resistance and failure of $\beta$-cell compensatory mechanisms, leads to increased metabolic fuels allowing excess nutrients to be transferred across the placenta to the fetus (1). Larger placentas are also more likely to occur in obese women, with altered levels of adipokines, which may also be associated with fetal growth $(1,23)$.

Gestational diabetes and obesity have similar metabolic and structural alterations and may affect fetal growth in similar ways. When combining maternal weight and GDM, we found that the two variables together have an even more pronounced effect on BW than either variable alone.

When examining the interaction effect between GDM and maternal weight, we found that lean women affected with GDM gave birth to babies with less weight in relation to both the $\mathrm{HC}$ and $\mathrm{BL}$ compared to controls. We hypothesize that the insulin treatment given to lean women with GDM may have a greater effect on growth potential, and in comparison to obese women treated with insulin, lean women might not have been able to compensate for low blood glucose values with higher lipid values, which have also been found to affect fetal growth (20). In this context, treatment with insulin would be interesting to analyze in future studies.

Previous studies have investigated the independent effect of GDM on other growth parameters, such as HC and BL. However, these studies have either examined growth parameters during pregnancy only or analyzed the odds ratio of LGA in length among babies born to women with $\operatorname{GDM}(16,24)$.
We found that the interaction between GDM and maternal BMI did not have a positive effect on BL or HC. Neither did GDM alone affect the HC. Nevertheless, GDM had an independent effect on BL.

\section{CONCLUSION}

Gestational diabetes increases the association between maternal weight and neonatal BW. In addition, GDM together with maternal BMI affects the neonatal growth patterns more than either variable alone. We hypothesize the variation in $\mathrm{BW}$, in relation to maternal BMI, contributes to the asymmetry among babies born to GDM women. How GDM in combination with maternal BMI and their effect on fetal growth patterns affect the well-being of the child later in life will need to be addressed in future studies.

\section{AUTHOR CONTRIBUTIONS}

All authors were involved in formulating the hypothesis and interpreting the data. All authors were involved in writing, editing, and approving the final manuscript. All authors agree to be accountable for all aspects of the manuscript.

\section{ACKNOWLEDGMENTS}

We would like to thank Janet Long for her assistance with the obstetrics database.

\section{REFERENCES}

1. King JC. Maternal obesity, metabolism, and pregnancy outcome. Annu Rev Nutr (2006) 26:271-91. doi:10.1146/annurev.nutr.24.012003.132249

2. Roseboom TJ, van der Meulen JH, Ravelli AC, Osmond C, Barker DJ, Bleker OP. Effects of prenatal exposure to the Dutch famine on adult disease in later life: an overview. Mol Cell Endocrinol (2001) 185:93-8. doi:10.1016/S0303-7207(01) 00721-3

3. de Boo H, Harding J. The developmental origins of adult disease (Barker) hypothesis. Aust N Z J Obstet Gynaecol (2006) 46:4-14. doi:10.1111/j.1479828X.2006.00506.x

4. Paneth N, Susser M. Early origin of coronary heart disease (the "Barker hypothesis”). BMJ (1995) 310:411-2. doi:10.1136/bmj.310.6977.411

5. Metzer BE, Lowe LP, Dyer AR, Trimble ER, Chaovarindr U, Coustan DR, et al. Hyperglycemia and adverse pregnancy outcomes. N Engl J Med (2008) 358:1991-2002. doi:10.1056/NEJMoa0707943

6. Oliveira D, Pereira J, Fernandes R. Metabolic alterations in pregnant women: gestational diabetes. J Pediatr Endocrinol Metab (2012) 25:835-42. doi:10.1515/ jpem-2012-0175

7. Kaaja R, Rönnemaa T. Gestational diabetes: pathogenesis and consequences to mother and offspring. Rev Diabet Stud (2008) 5:194-202. doi:10.1900/RDS. 2008.5.194

8. HAPO Study Cooperative Research Group. Hyperglycaemia and adverse pregnancy outcome (HAPO) study: associations with maternal body mass index. BJOG (2010) 117:575-84. doi:10.1111/j.1471-0528.2009.02486.x

9. Catalano PM, Ehrenberg HM. The short- and long-term implications of maternal obesity on the mother and her offspring. BJOG (2006) 113:1126-33. doi:10.1111/j.1471-0528.2006.00989.x

10. Australian Bureau of Statistics (ABS). Overweight and Obesity in Adults, Australia 2011-2012. (2013). Available from: http://www.abs.gov.au/ausstats

11. World Health Organization (WHO). BMI Classification. (2013). Available from: http://apps.who.int/bmi/

12. Flack JR, Ross GP, Ho S, McElduff A. Recommended changes to diagnostic criteria for gestational diabetes: impact on workload. Aust N Z J Obstet Gynaecol (2010) 50:439-43. doi:10.1111/j.1479-828X.2010.01218.x

13. Mongelli M, Figueras F, Francis A, Gardosi J. A customized birthweight centile calculator developed for an Australian population. Aust N Z J Obstet Gynecol (2007) 47:128-31. doi:10.1111/j.1479-828X.2007.00698.x

14. Langdon M, Gabbe S. Gestational diabetes mellitus. Obstet Gynecol (2011) 118:1379-93. doi:10.1097/AOG.0b013e31823974e2 
15. Ouzounian JG, Hernandez GD, Korst LM, Montoro MM, Battista LR, Walden $\mathrm{CL}$, et al. Pre-pregnancy weight and excess weight gain are risk factors for macrosomia in women with gestational diabetes. J Perinatol (2011) 31:717-21. doi:10.1038/jp.2011.15

16. Ahlsson F, Lundgren M, Tuvemo T, Gustafsson J, Haglund B. Gestational diabetes and offspring body disproportion. Acta Paediatr (2010) 99:89-93. doi:10.1111/j.1651-2227.2009.01532.x

17. Makgoba M, Savvidou MD, Steer PJ. The effect of maternal characteristics and gestational diabetes on birthweight. BJOG (2012) 119:1091-7. doi:10.1111/j. 1471-0528.2012.03388.x

18. Catalano PM, McIntyre HD, Cruickshank JK, McCance DR, Dyer AR, Metzger $\mathrm{BE}$, et al. The hyperglycemia and adverse pregnancy outcome study: associations of GDM and obesity with pregnancy outcomes. Diabetes Care (2012) 35:780-6. doi: $10.2337 / \mathrm{dc} 11-1790$

19. Catalano PM, Hauguel-De Mouzon S. Is it time to revisit the Pedersen hypothesis in the face of the obesity epidemic? Am J Obstet Gynecol (2011) 204:479-87. doi:10.1016/j.ajog.2010.11.039

20. Herrera E, Ortega-Senovilla H. Disturbances in lipid metabolism in diabetic pregnancy - are these the cause of the problem? Best Pract Res Clin Endocrinol Metab (2010) 24:515-25. doi:10.1016/j.beem.2010.05.006

21. Jansson T, Cetin I, Powell TL, Desoye G, Radaelli T, Ericsson A, et al. Placental transport and metabolism in fetal overgrowth - a workshop report. Placenta (2006) 27:109-13. doi:10.1016/j.placenta.2006.01.017

22. Larqué E, Ruiz-Palacios M, Koletzko B. Placental regulation of fetal nutrient supply. Curr Opin Clin Nutr Metab Care (2013) 16:292-7. doi:10.1097/MCO. 0b013e32835e 3674
23. Higgins L, Greenwood SL, Wareing M, Sibley CP, Mills TA. Obesity and the placenta: a consideration of nutrient exchange mechanisms in relation to aberrant fetal growth. Placenta (2011) 32:1-7. doi:10.1016/j.placenta.2010.09.019

24. Lampl M, Jeanty P. Exposure to maternal diabetes is associated with altered fetal growth patterns: a hypothesis regarding metabolic allocation to growth under hyperglycemic-hypoxemic conditions. Am J Hum Biol (2004) 16:237-63. doi:10.1002/ajhb.20015

Conflict of Interest Statement: The authors declare that the research was conducted in the absence of any commercial or financial relationships that could be construed as a potential conflict of interest.

Received: 19 June 2014; accepted: 01 October 2014; published online: 17 October 2014. Citation: Byström M, Liu A, Quinton AE, Champion BL, Mann K, Peek Mand Nanan RKH (2014) Gestational diabetes independently increases birth length and augments the effects of maternal BMI on birth weight: a retrospective cohort study. Front. Pediatr. 2:112. doi: $10.3389 /$ fped.2014.00112

This article was submitted to Neonatology, a section of the journal Frontiers in Pediatrics.

Copyright (C) 2014 Byström, Liu, Quinton, Champion, Mann, Peek and Nanan. This is an open-access article distributed under the terms of the Creative Commons Attribution License (CC BY). The use, distribution or reproduction in other forums is permitted, provided the original author(s) or licensor are credited and that the original publication in this journal is cited, in accordance with accepted academic practice. No use, distribution or reproduction is permitted which does not comply with these terms. 